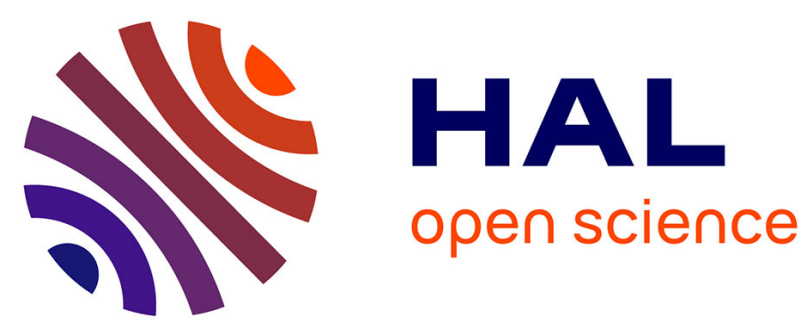

\title{
Relation entre composition salivaire, obésité et métabolisme lipidique postprandial chez l'Homme
}

Cécile Vors, Jocelyne Drai, Laure Gabert, Martine Laville, Elisabeth Guichard, Hélène Brignot, Marie-Caroline Michalski, Gilles Feron

\section{To cite this version:}

Cécile Vors, Jocelyne Drai, Laure Gabert, Martine Laville, Elisabeth Guichard, et al.. Relation entre composition salivaire, obésité et métabolisme lipidique postprandial chez l'Homme. 12. Journées Francophones de Nutrition (JFN), Dec 2015, Montpellier, France. hal-01595299

\section{HAL Id: hal-01595299 \\ https://hal.science/hal-01595299}

Submitted on 5 Jun 2020

HAL is a multi-disciplinary open access archive for the deposit and dissemination of scientific research documents, whether they are published or not. The documents may come from teaching and research institutions in France or abroad, or from public or private research centers.
L'archive ouverte pluridisciplinaire HAL, est destinée au dépôt et à la diffusion de documents scientifiques de niveau recherche, publiés ou non, émanant des établissements d'enseignement et de recherche français ou étrangers, des laboratoires publics ou privés. 


\section{Marseille \\ Parc Chanot \\ 9 - 11 décembre 2015}

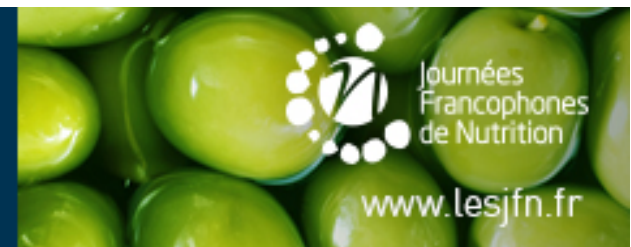

\section{Obésité}

JFN2015/ABS-1113

\section{Relation entre composition salivaire, obésité et métabolisme lipidique postprandial chez}

\section{l'Homme.}

Cécile Vors 1,2 , Jocelyne Drai ${ }^{1}, 2$, Laure Gabert ${ }^{1,2}$, Martine Laville ${ }^{1,2}$, Elisabeth Guichard ${ }^{3}$, Hélène Brignot ${ }^{3}$, Marie-Caroline Michalski ${ }^{1,2}$, Gilles Feron ${ }^{*} 3$

1INRA UMR1397, INSERM U1060, Univ. Lyon-1, Laboratoire CarMeN, Oullins, ${ }^{2}$ Centre de Recherche en Nutrition Humaine Rhône-Alpes (CRNH-RA) and Centre Européen pour la Nutrition et la Santé (CENS), Pierre-Bénite, ${ }^{3}$ CSGA, INRA, Dijon Cedex, France

Veuillez choisir votre discipline: Expérimental/mécanismes cellulaires et moléculaires

Présentation préférée: Poster

Spécifiez votre âge: 51-60 ans

Spécifiez votre lieu d'exercice: Chercheur

Introduction et but de l'étude: En situation d'obésité, l'exposition par voie orale aux matières grasses alimentaires peut moduler la digestion et l'absorption des lipides. Cependant les mécanismes oraux sous-jacents n'ont pas été clairement identifiés. Nous avons testé l'hypothèse que des composants de la salive impliqués dans la sensibilité orale aux lipides alimentaires seraient différents selon l'IMC et le métabolisme lipidique postprandial des individus.

Matériel et méthodes: La salive a été recueillie à jeun chez 9 sujets normo-pondérés (IMC $\left.=22,3 \pm 0,5 \mathrm{~kg} / \mathrm{m}^{2}\right)$ et 9 obèses (IMC=31,7 $\pm 0,3 \mathrm{~kg} / \mathrm{m}^{2}$ ) pour la mesure des paramètres suivants : flux, teneur en protéines, lipolyse, amylase, protéolyse, pouvoir antioxydant, lysozyme, teneurs en lipocaline 1 et en anhydrase carbonique- $\mathrm{VI}(\mathrm{CAVI})$. Ce recueil a été réalisé avant la consommation d'un repas contenant $40 \mathrm{~g}$ de matière grasse, suivie d'un test d'exploration métabolique de $8 \mathrm{~h}$, au cours duquel les sujets obèses ont révélé un métabolisme lipidique postprandial retardé.

Résultats et Analyse statistique: Nous montrons que la lipolyse salivaire est plus faible chez les sujets obèses par rapport aux normo-pondérés, alors que la protéolyse et la teneur en CAVI sont plus élevées. Par analyse multivariée et par test de Mann-Whitney, nous observons que ces différences de lipolyse et de protéolyse sont liées à des différences de métabolisme lipidique incluant l'absorption postprandiale des acides gras exogènes et leur bêta-oxydation.

Tableau: Variables discriminantes de composition salivaire et de métabolisme postprandial issues de l'analyse multivariée.

\begin{tabular}{|c|c|c|c|c|c|c|}
\hline & $\begin{array}{l}\text { Lipolyse } \\
(\mathrm{mU} / \mathrm{mL})\end{array}$ & $\begin{array}{l}\text { Protéolyse } \\
(\mathrm{U} / \mathrm{mL})\end{array}$ & $\begin{array}{l}\text { CAVI } \\
(\mathrm{ng} / \mathrm{ml})\end{array}$ & 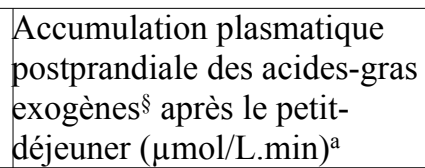 & $\begin{array}{l}{ }^{13} \mathrm{CO}_{2} \text { expiré après le petit- } \\
\text { déjeuner }(\% \cdot \mathrm{min})^{\mathrm{b}}\end{array}$ & $\begin{array}{l}\text { Accumulation postprandiale } \\
\text { des FRCM-TG après le } \\
\text { déjeuner }(\mathrm{mmol} / \mathrm{L} . \mathrm{min})^{\mathrm{c}}\end{array}$ \\
\hline $\begin{array}{l}\text { Normo- } \\
\text { pondéré } \\
\text { s }\end{array}$ & $0,25 \pm 0,07$ & $2 \pm 0,25$ & $140 \pm 47$ & $5,87 \pm 1,3$ & $2,9 \pm 0,25$ & $51 \pm 13$ \\
\hline Obèses & $0,12 \pm 0,02$ & $3,94 \pm 1$ & $260 \pm 55$ & $2,45 \pm 0,43$ & $1,56 \pm 0,23$ & $108 \pm 18$ \\
\hline P value & 0,036 & 0,03 & 0,07 & 0,045 & 0,018 & 0,036 \\
\hline
\end{tabular}

Valeurs moyennes $\pm E S M$. CAVI : anhydrase carbonique $\mathrm{VI}$; FRCM: fraction riche en chylomicrons ; ASCi: aire sous la courbe incrémentale ; TG: triglycérides.

a,b Calculé en aire sous courbe incrémentale entre 0 et 300 min après le petit-déjeuner, ${ }^{\circ}$ Calculé en aire sous courbe incrémentale entre 300 et 480 min après le petit-déjeuner (période post-déjeuner), §oléate et palmitate traceurs marqués au carbone 13. 
Conclusion: Ces résultats suggèrent un rôle de la salive sur le métabolisme lipidique postprandial. Ils amènent de nouvelles hypothèses causales sur les liens entre la composition de la salive, la sensibilité orale aux graisses alimentaires et le métabolisme lipidique postprandial en fonction de I'IMC.

\section{Références:}

1. Vors, C., G. Pineau, L. Gabert, J. Drai, C. Louche-Pelissier, C. Defoort, D. Lairon, M. Desage, S. Danthine, S. LambertPorcheron, H. Vidal, M. Laville, and M. C. Michalski. 2013. Am. J. Clin. Nutr. 97: 23-36.

2. Vors, C., J. Drai, L. Gabert, G. Pineau, M. Laville, H. Vidal, E. Guichard, M.-C. Michalski,and G. Feron. 2015. Int. J. Obes. doi: 10.138/ijo.2015.71.

Conflits d'intérêts: Aucun conflit à déclarer 